\title{
Diffusion Tensor Imaging of Cognitive Impairment Caused by Vascular Leukoencephalus Loosening
}

Wuqing Zhang ${ }^{1}$, Jing $\mathrm{Li}^{1}$, Xiaohong $\mathrm{Li}^{2}$ and Yifeng $\mathrm{Du}^{3 *}$

${ }^{1}$ Department of Neurology, Shanxian Central Hospital of Shandong Province, Shanxian, China

${ }^{2}$ Department of Neurology, Jinan Central Hospital Affiliated to Shandong University, Jinan, China

${ }^{3}$ Department of Neurology, Shandong Provincial Hospital Affiliated to Shandong University, Jinan, China

\begin{abstract}
Aim: Diagnosis and classification of Vascular Cognitive Impairment $(\mathrm{VCl})$ are still based on clinical manifestations and neuropsychological scales, but is not conducive to early clinical diagnosis and prevention. We aimed to investigate Diffusion Tensor Imaging (DTI) of cognitive impairment caused by vascular leukoencephalus loosening.
\end{abstract}

Methods: Using magnetic resonance diffusion tensor imaging DTI as a research method, in combination with neuropsychological testing, we investigated the relationship between brain white matter microstructural changes and cognitive impairment in patients with cognitive impairment. Systematic analysis was performed in all 60 patients with leukoaraiosis.

Results: Compared with the Normal Control (NC) group, the anisotropy fraction AF values in the Regions of Interest (ROIs) of vascular dementiaVaD patients were significantly decreased. The ADC values of the various regions of interest in vascular dementia $\mathrm{VaD}$ patients were significantly higher. anisotropy fraction AF values and apparent diffusion coefficient $A D C$ values and cognitive scores in specific white matter areas were found to be independent predictors in non-dementia vascular cognitive impairment VCIND.

Conclusion: The relationship between changes in white matter microstructure and cognitive function provides the basis for clinical control and ideas for early detection, early prevention and early treatment.

Keywords: Leukoaraiosis; Alzheimer's disease; Dementia

Abbreviations: VCI: Vascular Cognitive Impairment; VCIND: Non-dementia Vascular Cognitive Impairment; DTI: Diffusion Tensor Imaging; AF: Anisotropy Fraction; ADC: Apparent Diffusion Coefficient; VaD: Vascular Dementia

\section{Introduction}

According to a recent report in The Daily Telegraph, more than 70,000 British people died of Alzheimer's disease and dementia every year and 66,000 people died of heart disease. Dementia first surpassed heart disease and became the number one killer in the UK [1]. With the acceleration of the global aging process, the incidence of cerebrovascular dementia is on the rise, which seriously affects the patient's ability to adapt to the society. It is also an important unfavorable factor affecting the comprehensive recovery of cerebrovascular disease. Vascular Cognitive Impairment (VCI) refers to impairment of cognitive function caused by or associated with vascular factors. VCI is a type of syndrome from mild cognitive impairment to dementia [2]. As with Alzheimer's disease, advancing age is a major risk factor for VCI or dementia. Additional risk factors that affect blood vessels may raise risk for heart problems, stroke and other diseases. VCI includes two important stages of Vascular Dementia (VaD) and Non-dementia Vascular Cognitive Impairment (VCIND) [3]. Vascular dementia, the second most common form of dementia in older adults after Alzheimer's disease and VCI results from injuries to cerebral blood vessels after a stroke. In the early period, about $50 \%$ of VCIND patients will progress to dementia within 5 years [4].

The degree of cognitive impairment is light and early reversibility is the only dementia disease that can be prevented [5]. Unfortunately, due to the lack of uniform and satisfactory diagnostic criteria, the research progress of VCI has been greatly hindered. The focus of the study is gradually turning to cerebral small vessel disease. It has been reported that the incidence of white matter osteoporosis in the elderly is as high as $50 \%-98 \%$. Cerebral small vessel disease-related cognitive impairment accounted for about $50 \%$ of VCI patients [6].

At present, the diagnosis and classification of VCI is still based on clinical manifestations, neuropsychological scales and subjective, but is not conducive to early clinical diagnosis and prevention [7]. In 2011, the Dementia and Cognitive Disorders Group of the Chinese Medical Association Neurology Branch proposed a consensus for the diagnosis of non-dementia vascular cognitive impairment with VCIND [8]. It also emphasized neuroimaging. The examination is an important means to identify and diagnose VCIND. Early detection, early diagnosis and early intervention of VCI become possible to a certain extent. Recognition and intervention of VCI patients as soon as possible will greatly improve the prognosis of patients.

For cognitive dysfunction, abnormal changes in tissue function generally precede changes in the structure. Prior to significant structural changes in imaging, the brain tissue structure may have shown functional abnormalities. Although neuroimaging plays an important role in the evaluation of cerebrovascular diseases in patients with VCI, structural imaging parameters have obvious lag in the

*Corresponding author: Dr. Yifeng Du, Department of Neurology, Shandong Provincial Hospital Affiliated to Shandong University, Jinan, China, Tel: +8613583118172; E-mail: 2945751942@qq.com

Received December 27, 2018; Accepted January 10, 2019; Published January 15,2019

Citation: Zhang W, Li J, Li X, Du Y (2019) Diffusion Tensor Imaging of Cognitive Impairment Caused by Vascular Leukoencephalus Loosening. J Neurol Neurophysiol 10: 481. doi:10.4172/2155-9562.1000481

Copyright: $\odot 2019$ Zhang W, et al. This is an open-access article distributed under the terms of the Creative Commons Attribution License, which permits unrestricted use, distribution, and reproduction in any medium, provided the original author and source are credited. 
diagnosis of VCI. Routine examinations such as CT and MRI routine sequences (T1, T2, DWI and FLAIR sequences) provide only a little information for potential white matter-transmitted bundles and have limited clinical diagnostic value. Diffusion Tensor Imaging (DTI) is an imaging method that uses the diffusion motion anisotropy of water molecules to sensitively reflect the structural changes of white matter fibers in the brain and to detect the exchange of water molecules between various components in the pathological state of living tissue [9]. At present, there are few researches on the correlation between the early diagnosis of VCI and the degree of disease in DTI imaging.

\section{Object and Methods}

\section{Object data}

Continuous selection of patients with vascular leukoaraiosis in our hospital for the first time from July 2015 to April 2016 was performed. All patient recruitment was approved by the Research Bioethical Committee in the hospitals involved in this study. There were 30 patients with dementia-type VCI and 30 patients with vascular dementia. Another 30 healthy volunteers were selected for diagnosis and classification of VCI. Referring to the standards proposed by the National Institute of Neurological Disorders and Stroke (NINDS) and the Canadian Stroke Network (CSN) in the United States in 2006 [10], the Chinese versions of both the Montreal Cognitive Assessment Scale (MoCA) and the Clinical Dementia Rating Scale (CDR) were used. Activity of Daily Living scale (ADL) and Hachinski Ischemic Score (HIS) were tested. The study obtained the right of informed consent, excluding the enhancement of white matter signal caused by non-vascular factors and excluding the history of cognitive disorders caused by other definite causes, such as Alzheimer's disease, Parkinson's disease and normal intracranial pressure hydrocephalus. And hypothyroidism; there are contraindications such as magnetic resonance imaging. All subjects were diagnosed by 2 deputy chief physicians and above neurological physicians based on medical history, symptoms, neurological examinations, routine laboratory tests and plain MRI (Medical Resonance Imaging) scans.

In the dementia-free VCI group, there were 18 males and 12 females, aged 58 to 83 years, mean $(66.5 \pm 14.2)$ years, history of stroke 12 cases, history of hypertension 8 cases, history of diabetes 6 cases and history of heart disease 5 cases. There were 8 cases of hyperlipidemia and 11 cases of smoking. The average level of education was (10.8 $\pm+/$ 3.6) years, Mo CA average score was $(22.4 \pm 4.5)$ points, CDR average score was less than 1.0, ADL average score was $(21.5 \pm 6.6)$ points and HIS average. The score was $(5.8 \pm 1.4)$ points. In the vascular dementia group, there were 16 males and 14 females, aged $56-85$ years, mean $(64.8 \pm 15.6)$ years old, including 13 patients with stroke history, 7 with hypertension, 8 with diabetes and 4 with heart disease history. There were 8 cases of hyperlipidemia history and 10 cases of smoking. The average level of education was $(11.2 \pm 3.8)$ years. The average scores include MoCA (18.6 \pm 4.4$)$, CDR $(1.7 \pm 0.5)$ and ADL (33.8 \pm 7.2$)$, respectively. The average HIS score was $(8.5 \pm 2.3)$ points. The control group included 15 males and 15 females, aged 55-82 years, mean ( 65.8 \pm 15.5 ) years, history of stroke 10 , history of hypertension, 7 cases of diabetes, 4 history of heart disease and history of hyperlipidemia 6 . For example, smoking was found in 9 cases. The average level of education was $(11.3 \pm 4.3)$ years and the average scores are: Mo CA (27.01 \pm 1.43$)$, $\mathrm{CDR}=0, \mathrm{ADL}<26$ and $\mathrm{HIS}=0$.

\section{Methods}

According to the MRI inclusion criteria, 60 patients with vascular
LA were included and 30 healthy and elderly patients with comparable age were normal controls. LA patients were divided into VD group 30 cases and VCIND group 30 cases according to the diagnostic criteria of cognitive impairment. Conducted neuropsychology tests include MiniMental State Examination (MMSE), MoCA and seven factors including memory, attention, language, visual space structure and executive function. The head DTI examination was performed to measure the Fraction Anisotropy Factor (FAF) and mean diffusivity of white matter (including lesions and normal parts) of the bilateral frontal lobe, temporal lobe, occipital lobe, semiovale center, lateral periventricular thalamus and thalamus, respectively. The differences in DTI parameters between the 3 groups were analyzed and correlation analysis between the VCIND group and multiple cognitive domain neuropsychological test results was performed.

\section{DTI imaging}

All subjects underwent cranial scan using a $3.0 \mathrm{~T}$ magnetic resonance imaging apparatus (GE SIGNA EXCITE II) and selected the head coil. DTI scan adopts axial SE EPI sequence, acquisition time is $6 \sim 7 \mathrm{~min}, \mathrm{TR}=6000 \mathrm{~ms}, \mathrm{TE}=90 \mathrm{~ms}$, layer thickness $3 \mathrm{~mm}$, layer number 45 , bandwidth $150 \mathrm{kHz}, \mathrm{FOV}=240 \mathrm{~mm} \times 240 \mathrm{~mm}$, matrix $128 \times 128$, $\mathrm{FAF}^{\circ}$, The average number of excitations was 3 and the $b$ value was 0 . The diffusion characteristics were measured along 20 non-collinear directions. In the 3D T1WI position on the sagittal plane, the baseline of the scan remained horizontal, covering the entire brain. The workstation DTI analysis software package (Neuro 3D) was used to reconstruct FAF maps, Apparent Diffusion Coefficient (ADC) maps and b0 maps. Red, blue and green on the FAF images represent the left, right, top and back, respectively. The regions of interest on different levels of the FAF axis bitmap were selected and the FAF values and the ADC values were measured. The voxels on both sides are basically symmetrical in position and size. Optimal levels were selected for analysis of the Region of Interest (ROI). The data were measured in white matter and normal white matter in the following regions: bilateral subfrontal cortex, temporal lobe, occipital lobe subcortical white matter, bilateral semispheric center, bilateral lateral ventricle, bilateral thalamus. Each ROI is located at the center of the largest display plane of the anatomy. For the frontal lobe, the middle gyrus and the parietal lobe take three angles each; the temporal lobe takes three successive layers after the lateral lobulation; the occipital lobe takes the occipital pole and takes two layers; the frontal and posterior horns of the lateral ventricle The body was positioned on two consecutive levels on the lateral or slightly inferior side of the lateral ventricle and placed approximately $3 \mathrm{~mm}$ outside the anterior and posterior horns of the lateral ventricle. Thalamus: The ROI of the elliptical map is placed at the maximum of $2 \mathrm{~mm}$ from the posterior branch of the internal capsule to the three consecutive levels of the anterior joint and below. The same anatomical structures of the bilateral cerebral hemispheres were selected using mirror symmetry. Each ROI was measured multiple times to ensure that the FAF value of the selected slice of the site was the largest and the left and right sides were measured 3 times. The results were expressed as the mean value of FAF and ADC on each side. The ROI area is 30-40 $\mathrm{mm}^{2}$. When placing the ROI, avoid hemorrhages, adjacent gray matter cortex, large blood vessels, ventricles, bones, sinus air and brain edge, gray matter structure and cerebrospinal fluid. When the area is difficult to define, refer to the same level of T2WI image to help define the scope of each area. When looking for white matter lesions, attention was paid to combining FLAIR and DWI images to exclude simultaneous infarct or brain soften lesions that existed at different times. The distance between the ROI of the lesion, the normal white matter and the edge of the lateral ventricle, the infarct and the gray matter is at least 2 
Citation: Zhang W, Li J, Li X, Du Y (2019) Diffusion Tensor Imaging of Cognitive Impairment Caused by Vascular Leukoencephalus Loosening. J Neurol Neurophysiol 10: 481. doi:10.4172/2155-9562.1000481

mm. Placement and measurement of all ROIs of the participants were performed by the same observer.

\section{Statistical method}

SPSS13.0 software was used for statistical analysis. When the measurement data satisfied the normal distribution and variance, the variance and FAF and ADC values of each ROI of the three groups were compared using variance analysis. When the normal distribution and variance were not satisfied, multiple sample rank sums were used. Tests were performed for statistics; according to different data, Pearson correlation analysis or Spearman correlation analysis was used to count correlations between FAF and ADC values and cognitive scores at each site. Normally measured data were expressed as mean \pm standard deviation $(\mathrm{x} \pm \mathrm{s})$ and skewed distribution data were expressed as median (interquartile range) $[\mathrm{Md}(\mathrm{QR})] . \mathrm{p}<0.05$ was considered statistically significant.

\section{Results}

\section{Changes in FAF values of brain regions in three groups of subjects}

The FAF values of the brain regions of all three groups were

\begin{tabular}{|c|c|c|c|c|c|}
\hline \multirow{2}{*}{ Group } & \multicolumn{4}{|c|}{ Anisotropy Fraction (AF) } \\
\cline { 2 - 6 } & VCIND & VaD & NC & $\begin{array}{r}\mathbf{F} \\
\text { value }\end{array}$ & $\begin{array}{c}\mathbf{p} \\
\text { value }\end{array}$ \\
\hline Frontal lobe & $0.48 \pm 0.15^{*}$ & $0.36 \pm 0.09^{\star \Delta}$ & $0.76 \pm 0.23$ & 5.632 & 0.020 \\
\hline Temporal lobe & $0.52 \pm 0.16^{*}$ & $0.39 \pm 0.11^{\star \Delta}$ & $0.83 \pm 0.25$ & 5.527 & 0.023 \\
\hline Occipital lobe & $0.35 \pm 0.15^{*}$ & $0.22 \pm 0.04^{\star \Delta}$ & $0.64 \pm 0.25$ & 5.314 & 0.026 \\
\hline $\begin{array}{c}\text { Centrum } \\
\text { semiovale }\end{array}$ & $0.50 \pm 0.21^{*}$ & $0.35 \pm 0.12^{\star \Delta}$ & $0.84 \pm 0.28$ & 5.867 & 0.017 \\
\hline Side of ventricle & $0.48 \pm 0.16^{*}$ & $0.35 \pm 0.11^{\star \Delta}$ & $0.79 \pm 0.22$ & 6.132 & 0.012 \\
\hline Thalamus & $0.44 \pm 0.17^{*}$ & $0.25 \pm 0.06^{\star \Delta}$ & $0.83 \pm 0.27$ & 6.157 & 0.015 \\
\hline
\end{tabular}

Table 1: Changes of FAF values (units) in each brain area of three groups of subjects (Note: *- indicates $p<0.05$ compared with NC group; $\Delta$ indicates $p<0.05$ compared with VCIND group).

\begin{tabular}{|c|c|c|c|c|c|}
\hline \multirow{2}{*}{ Group } & \multicolumn{5}{|c|}{ ADC $\left(\times \mathbf{1 0}^{-\mathbf{3}} \mathbf{m m}^{\mathbf{2}} / \mathbf{s}\right)$} \\
\cline { 2 - 6 } & VCIND & VaD & NC & F value & p value \\
\hline Frontal lobe & $0.57 \pm 0.12^{*}$ & $0.86 \pm 0.25^{\star \Delta}$ & $0.32 \pm 0.09$ & 6.325 & 0.013 \\
\hline Temporal lobe & $0.46 \pm 0.11^{*}$ & $0.77 \pm 0.19^{\star \Delta}$ & $0.35 \pm 0.06$ & 6.217 & 0.016 \\
\hline Occipital lobe & $0.47 \pm 0.12^{*}$ & $0.80 \pm 0.23^{\star \Delta}$ & $0.26 \pm 0.05$ & 6.655 & 0.007 \\
\hline Centrum semiovale & $0.35 \pm 0.09^{*}$ & $0.69 \pm 0.16^{\star \Delta}$ & $0.19 \pm 0.03$ & 6.358 & 0.011 \\
\hline Side of ventricle & $0.36 \pm 0.06^{*}$ & $0.66 \pm 0.14^{\star \Delta}$ & $0.23 \pm 0.04$ & 6.753 & 0.005 \\
\hline Thalamus & $0.34 \pm 0.06^{*}$ & $0.75 \pm 0.18^{\star \Delta}$ & $0.16 \pm 0.06$ & 6.968 & 0.002 \\
\hline
\end{tabular}

Table 2: Table of Changes in ADC Values in Brain Regions of Three Groups of Subjects (Note: *-indicates $p<0.05$ compared with NC group; $\Delta$ indicates $p<0.05$ compared with VCIND group). comparable, with statistical differences $(\mathrm{P}<0.05)$. Comparison between the two groups: Compared with the Normal Control (NC) group, the FAF values in the ROIs of VaD patients were significantly decreased $(\mathrm{P}<0.05)$; the FAF values in the ROIs of the patients in the VCIND group were decreased $(\mathrm{P}<0.05)$; the patients in the $\mathrm{VaD}$ group and the VCIND group were compared. There were statistically significant differences $(\mathrm{P}<0.05)$ (Table 1$)$.

\section{Changes of ADC values in brain regions of three groups of subjects}

Statistical analysis showed that the three sets of data were overall comparable, with statistical differences $(\mathrm{p}<0.05)$ and the pairwise comparison: Compared with the NC group, the ADC values of the various regions of interest in $\mathrm{VaD}$ patients were significantly higher $(p<0.05)$. The ADC value in each region of interest in the VCIND group was significantly increased $(\mathrm{p}<0.05)$; in the $\mathrm{VaD}$ group and the VCIND group: ADC values were statistically different in each region of interest $(\mathrm{p}<0.05)$ (Table 2).

\section{Correlation analysis of FAF value, ADC value and neuropsychological score in each brain area}

In the control of age and education, aside white matter lesions and the impact of $\mathrm{VaD}$ patient scores, the correlation between FAF values and ADC values and cognitive scores in specific white matter areas was studied in VCIND, which were found to be independent predictors. The specific data are shown in Tables 3 and 4 .

From the table, we can see that controlling the age and degree of education, aside the white matter lesions and $\mathrm{VaD}$ patient scores, the frontal lobe FAF value of the subjects in the visual space and the ability to perform, attention, abstract has a statistically significant difference. Independently significant correlations were observed; FAF values of temporal lobe were correlated in visual space and executive ability, naming and memory; FAF values in occipital lobe had strong independent correlation in visual space, executive ability and attention; half-oval center FAF values. It has a strong correlation with the total score and attention and it has a strong correlation with abstract power. The FAF of the lateral ventricle is independently related to the total score, visual space, executive ability and abstract power and has strong memory and attention. Relevance and thalamic FAF values have strong correlations depending on space and execution ability, attention and abstraction.

From the table, we can see that controlling the age and level of education, leaving the impact of $\mathrm{VaD}$ patient scores, VCIND subject frontal lobe $\mathrm{ADC}$ values have independent significant correlation in the visual space and execution ability and scores total score, attention, abstract There was significant statistical difference and correlation;

\begin{tabular}{|c|c|c|c|c|c|c|}
\hline Cognitive scores & Frontal lobe & Temporal lobe & Occipital lobe & Centrum semiovale & Side of ventricle & Thalamus \\
\hline MMSE & $0.385^{*}$ & 0.234 & 0.256 & $0.456^{* *}$ & $0.564^{\star *}$ & 0.321 \\
\hline MOCA & $0.398^{*}$ & 0.265 & 0.286 & $0.487^{* *}$ & $0.579^{* *}$ & 0.332 \\
\hline Executive & $0.575^{* *}$ & $0.385^{*}$ & $0.312^{*}$ & $0.456^{* *}$ & $0.512^{* *}$ & $0.315^{*}$ \\
\hline Denominate & 0.254 & $0.372^{*}$ & 0.231 & 0.287 & 0.213 & 0.198 \\
\hline Memory & 0.233 & $0.352^{*}$ & 0.282 & 0.374 & $0.318^{*}$ & 0.223 \\
\hline Attention & $0.421^{\star *}$ & 0.278 & $0.327^{*}$ & $0.405^{\star *}$ & $0.327^{*}$ & $0.307^{*}$ \\
\hline Language & 0.157 & 0.239 & 0.023 & 0.197 & 0.210 & 0.115 \\
\hline Abstract & $0.412^{* *}$ & 0.279 & 0.298 & $0.347^{*}$ & $0.412^{\star *}$ & $0.352^{*}$ \\
\hline Orientation & 0.163 & 0.175 & 0.145 & 0.101 & 0.021 & 0.054 \\
\hline
\end{tabular}

Table 3: Pearson correlation analysis of FAF values and neuropsychological scores in each brain region of VCIND (Note: Correlation analysis between the two groups, * $p<0.05 ;{ }^{* *}: p<0.01$, statistically significant). 
Citation: Zhang W, Li J, Li X, Du Y (2019) Diffusion Tensor Imaging of Cognitive Impairment Caused by Vascular Leukoencephalus Loosening. J Neurol Neurophysiol 10: 481. doi:10.4172/2155-9562.1000481

\begin{tabular}{|c|c|c|c|c|c|c|}
\hline Cognitive scores & Frontal lobe & Temporal lobe & Occipital lobe & Centrum semiovale & Side of ventricle & Thalamus \\
\hline MMSE & $-0.341^{*}$ & -0.213 & -0.187 & $-0.372^{*}$ & $-0.384^{*}$ & -0.154 \\
\hline MOCA & $-0.357^{*}$ & -0.251 & -0.199 & $-0.381^{*}$ & $-0.393^{*}$ & -0.176 \\
\hline Executive & $-0.482^{* *}$ & $-0.315^{\star}$ & $-0.301^{*}$ & $-0.378^{*}$ & $-0.425^{\star *}$ & $-0.298^{*}$ \\
\hline Denominate & -0.212 & $-0.304^{*}$ & -0.119 & -0.216 & -0.187 & -0.165 \\
\hline Memory & -0.201 & $-0.302^{*}$ & -0.257 & -0.287 & -0.219 & -0.116 \\
\hline Attention & $-0.315^{\star}$ & -0.251 & -0.299 & $-0.357^{*}$ & $-0.312^{*}$ & -0.295 \\
\hline Language & -0.127 & -0.201 & -0.018 & -0.183 & -0.250 & -0.216 \\
\hline Abstract & $-0.314^{*}$ & -0.211 & -0.165 & $-0.302^{*}$ & $-0.334^{*}$ & -0.212 \\
\hline Orientation & -0.120 & -0.103 & -0.156 & -0.117 & -0.014 & -0.029 \\
\hline
\end{tabular}

Table 4: Pearson correlation analysis of ADC values and neuropsychological scores in each brain region of VCIND (Note: Correlation analysis between the two groups, *: $p<0.05 ;{ }^{* *}: p<0.01$, statistically significant).

temporal lobe ADC values depended on spatial and executive ability, naming and memory; occipital lobe ADC values depended on spatial and executive ability; half-oval central ADC values and score total scores, visual space and executive ability, attention and abstraction are independently related. ADC of the lateral periventricular ventricular region is independently related to the total score, attention and abstraction abilities. It is independently related to visual acuity and executive ability. The spatial value of the thalamus ADC value depends on Ability has a strong correlation. The above correlations are all negatively correlated.

\section{Discussion}

Leukoaraiosis (LA) is also called White Matter Lesions (WML) or White Matter Hyperintensity (WMH) is an imaging diagnostic term first proposed by Canadian neurologist Hachinski et al. [11] in 1987 and is a type of Cerebro-vascular Disease (CSVD) [12].

Diffusion tensor imaging is a new MR technique developed in recent years. It can use multiple different sizes of diffusion-sensitive gradients to show the strength of diffusion of water molecules in vivo and the directionality of diffusion motion. It is the only one so far, which is a non-invasive visualization of the diffusion characteristics of living tissue water molecules [13]. In the brain, the gray matter of the brain shows a slight anisotropy and the white matter of the brain shows large diffusion anisotropy because the myelin restricts the diffusion of water transversely through the axons. In the white matter of the brain, the dispersion of the vertical nerve fibres in the direction of travel will be limited by myelin and perineurium membranes, so the diffusion velocity in the direction of the vertical nerve fibres is less than that along the fibers, i.e., dispersion anisotropy $[14,15]$. DTI technology observes and measures the white matter fibres of the brain by reflecting the Brownian motion of water molecules. The microscopic level can reflect the tightness of fiber bundle arrangement, axonal membrane, axonal myelination degree, etc. and the macroscopic level can reflect the direction of fiber bundle travel [16]. The FA value is the ratio of water molecules anisotropy component to the whole diffusion tensor, which ranges from 0 to 1 . It approaches 1 and increases toward the opposite sex, indicating that the organization is arranged more closely and closer to 0 . Same-sex, poor organization regularity $[17,18]$ mainly reflects the integrity of nerve fiber anatomy. ADC is the average degree of diffusivity in all directions and it has no directionality; it reflects the diffusion area of water molecules per unit time and varies with the strength and duration of the applied diffusion gradient. ADC values reflect the diffusion rate and range of water molecules and quantify the integrity of the microstructure of the white matter fiber bundles [19], which mainly reflect the size of the extracellular space. The larger the ADC value, the weaker the molecular dispersion ability, reflecting the greater destruction of the integrity of the white matter fiber microstructure [20]. Combining these two parameters can accurately evaluate the fine structure changes of brain tissue, especially white matter.

DTI detection of changes in the white matter microarchitecture is inferior to conventional MRI. With the progression of the disease, when the conventional MRI parameters have not been detectably changed, DTI parameters (mainly FAF) have undergone detectable changes [21]. This change is consistent with age-related white matter changes in axonal loss and gliosis. Because the axon produces a significant water diffusion barrier, the water content within the tissue increases when the axon is absent and the degree of the barrier decreases, causing changes in DTI-related parameters. The high sensitivity of DTI to white matter suggests that it can serve as a good reference for disease progression and therapeutic effects. In this study, ROI selection was not only restricted to the white matter lesion area, but also included the normal white matter area. It was found that the cognitive impairment dynamically developed from normal to mild cognitive impairment, to cognitive blood vessels. In the development process of dementia, FAF values and ADC values in DTI have a good degree of discrimination in each stage of cognition. With the development of cognitive impairment, there is an increase in FAF value and a decrease in ADC value, with statistical differences. DTI can serve as an imaging marker for early cognitive impairment.

In this study, it was found that the change of two parameters of multiple ROIs in the case group was not parallel and the increase in ADC value was not consistent with the area in which the FAF value decreased. The reason may be that the former decreased more with neuronal loss and demyelination, while the latter increases in association with is related to the expansion of the extracellular space, The FAF value is more sensitive to the destruction of nerve fiber integrity. In At the early stage of cognitive impairment, micro-damage has already occurred in the fiber bundles and the loss of myelin sheath and axonal membrane has occurred. However, the relative macroscopic performance is not obvious. If the cell gap is not significantly expanded, the FAF value may have decreased and the ADC has increased. The height was not significant, but there was no significant difference between the ADC and the control group. In the index of anisotropy, the FAF value is widely used due to the following reasons: 1) The FAF value does not change with the change of the rotation direction of the coordinate system; 2) The FAF image can provide better gray matter contrast; 3) FAF map The signal-to-noise ratio is high; 4) FAF values are the physical characteristics of the organization and are comparable between the same object at different times, between different objects and between values obtained by different imaging devices. The above characteristics are destined to change the FAF values more sensitive than the ADC values. 
Citation: Zhang W, Li J, Li X, Du Y (2019) Diffusion Tensor Imaging of Cognitive Impairment Caused by Vascular Leukoencephalus Loosening. J Neurol Neurophysiol 10: 481. doi:10.4172/2155-9562.1000481
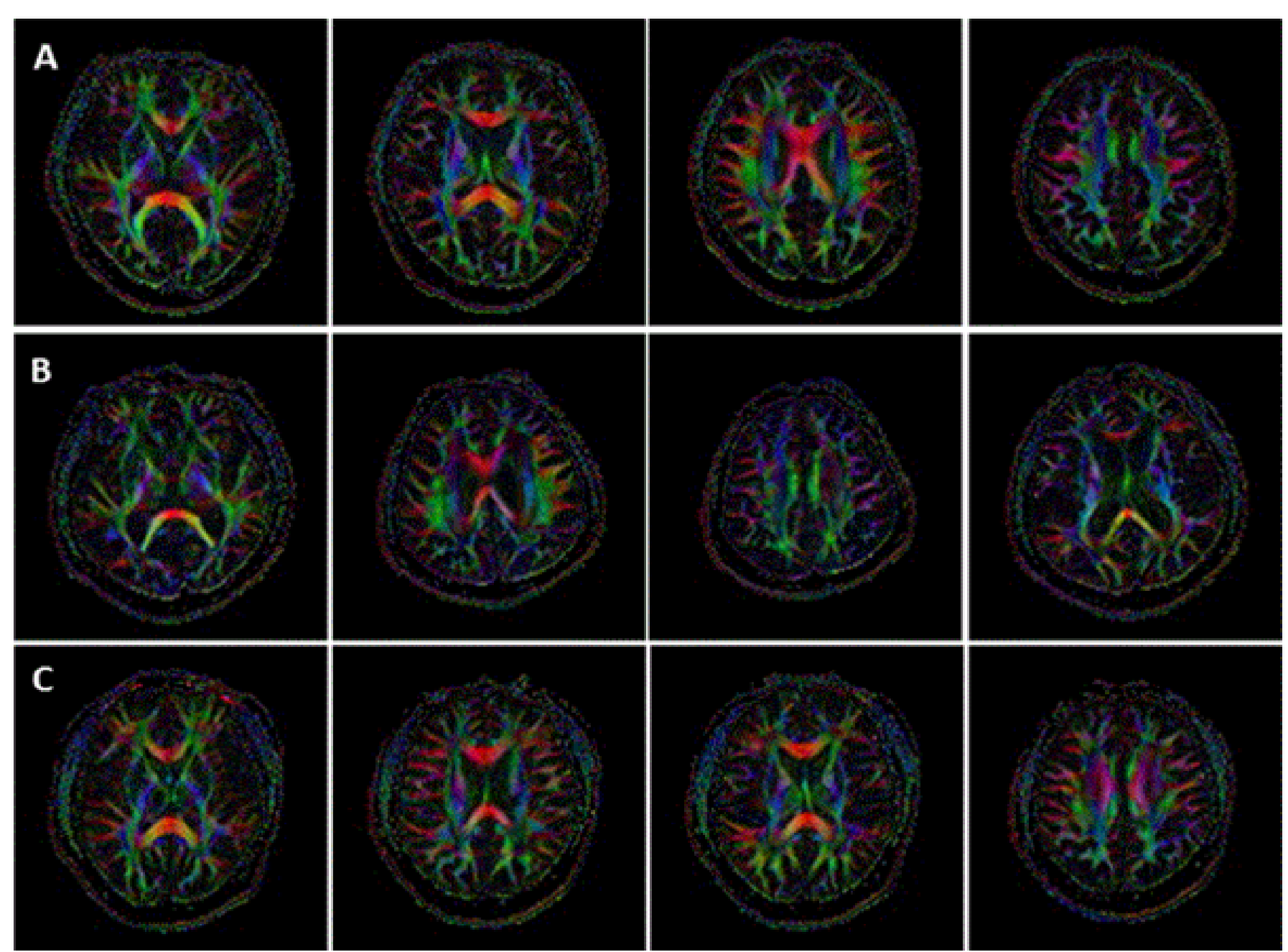

Figure 1: White matter fiber bundles detected by Diffusion Tensor Imaging (DTI) (A: Normal human white matter fiber bundles; B: Patients with vascular dementia (VaD); C: Patients with Non-dementia Vascular Cognitive Impairment (VCIND); Note: DTI studio software processes the color-coded FAF map to show the direction and shape of the fiber bundles).

The results of this study show that, compared with the normal control group, the ADC values of mild vascular cognitive impairment and vascular dementia syndrome are all increased, while the FAF value is decreased, suggesting that the degree of white matter fiber damage changes with the disease. Simply analyzing the VCI from the DTI we can see white matter structure and fiber damage, indicating that DTI is feasible in the detection of VCI. In VCIND, controlling the age and degree of education, aside the impact of $\mathrm{VaD}$ patient scores, the subject's FAF value, ADC value in the frontal lobe, temporal lobe, occipital lobe, semi-ovary center, lateral ventricle weeks, etc. Through a variety of cognitive function changes associated, but the function associated with each brain region is also different: for example, bilateral frontal lobe, occipital lobe, semi-ovary center, lateral periventricular, thalamus FAF values, ADC values and executive function correlations; FAF, ADC values of bilateral temporal correlates with naming and memory, FAF and ADC values of the lateral ventricle are correlated with attention and FAF, ADC values and abstract powers of the frontal lobe, lateral ventricle and semi-ovale correlations, etc., but the FAF, ADC values of each brain area are not related to language and orientation, suggesting that early language ability and orientation of cognitive impairment are rarely impaired, which is consistent with clinical manifestations. In At the early stage of vascular cognitive impairment, changes in execution ability, processing speed and decline in abstract thinking ability are mainly reflected in [22]. Further from the side, DTI can be used as an imaging predictor of early cognitive impairment. The disruption of the thalamus-basal ganglia-prefrontal cortex is the pathological anatomy underlying VCI cognitive impairment, behavioral abnormalities and other clinical symptoms $[23,24]$ (Figure 1).

\section{Conclusion}

In conclusion, our results showed that FAF decreased and ADC increased in multiple brain white matter normal areas in LA patients, suggesting that there was selective fine structure damage in white matter in LA; its changes were related to multiple aspects of cognitive impairment in LA, which further reflected the degree of decline in cognitive function. DTI can better compensate for the deficiency of conventional MRI and provide more detailed indicators for the damage of white matter. The use of neuropsychological examination combined with DTI research methods will help us improve our understanding of LA white matter lesions, observe dynamic changes during disease progression and gain a 
Citation: Zhang W, Li J, Li X, Du Y (2019) Diffusion Tensor Imaging of Cognitive Impairment Caused by Vascular Leukoencephalus Loosening. J Neurol Neurophysiol 10: 481. doi:10.4172/2155-9562.1000481

deeper understanding of white matter lesions and cognitive function in LA patients. The relationship between damage provides a theoretical basis for early clinical diagnosis and early intervention. We should also see that DTI imaging has some limitations, such as partial volume effect caused by too low resolution, turbulent effects caused by diffused gradient pulses and there is currently no gold standard for fiber bundle tracing. With the continuous advancement of MRI hardware and software, these problems will gradually be solved.

\section{References}

1. www.alzheimersresearchuk.org

2. Gorelick PB, Pantoni L (2013) Advances in vascular cognitive impairment. J Stroke 44: 307-308.

3. Singh $T$, Newman AB (2011) Inflammatory markers in population studies of aging. Ageing Res Rev 10: 319-329.

4. Jiang B, Ding C, Yao G (2013) Polysomnographic abnormalities in patients with vascular cognitive impairment-no dementia. J Sleep Med 14: 1071-1075.

5. Deborah A, Kenneth ML, Langa $L$ (2011) Vascular cognitive impairment: Disease mechanisms and therapeutic implications. Neurotherapeutics 8: 361-373.

6. Zhang AJ, YuXJ, Wang M (2010) The clinical manifestations and pathophysiology of cerebral small vessel disease. J Neurosci Bull 26: 257-264.

7. Jellinger AK (2013) Pathology and pathogenesis of vascular cognitive impairment-a critical update. Front Aging Neurosci 5: 17.

8. Chinese medical association neurology branch dementia and cognitive disorders group writing group (2011) Guidelines of vascular cognitive disorders diagnosis and treatment. Chinese $\mathrm{J}$ of Neurol pp: 2.

9. Zeestraten EA, Benjamin P, Lambert C, Lawrence AJ, Williams OA, et al. (2016) Application of diffusion tensor imaging parameters to detect change in longitudinal studies in cerebral small vessel disease. PLoS One 11: e0147836.

10. Hachinski V, ladecola C, Petersen RC (2006) National institute of neurological disorders and stoke-canadian stoke network vascular cognitive impairment harmonization standards. Stroke 37: 2220-2241.

11. Hachinski VC, Potter P, Merskey H (1987) Leukoaraiosis. J Arch Neurol 44: 21-26.
12. LADIS study group (2011) 2001-2011: A decade of the LADIS (Leukoaraiosis and Disability) study: What have we learned about white matter changes and small-vessel disease? J Cerebrovasc Dis 32: 577-588.

13. Hajnal JV, Doran M, Hall AS (1991) MR imaging of anisotropically restricted diffusion of water in the nervous system: technical, anatomic, andpathologic considerations. J Comput Assist Tomogr 15: 1-18.

14. Chang C, Li WB, Xie WH (2010) Diffusion tensor imaging of association fiber tracts in amnestic mild cognitive impairment. $\mathrm{J}$ Chinese $\mathrm{J}$ of Med Imaging Technol 26: 56-59.

15. Thomas C, Humphreys K, Jung KJ (2011) The anatomy of the callosal and visual association pathways in high functioning autism: A DTI tractography study. J Cortex 47: 863-873.

16. Park CH, Kou N, Boudrias MH (2013) Assessing a standardized approach to measuring corticospinal integrity after stroke with D. J Neuroimage Clin 2: 521 533.

17. Guo CP, Zhang XL, Chen ZP (2014) Diffusion tensor imaging anddiffusion tensor tractography in brainstem tumors with the mediallemnnicus invaded. Chinese J Neurosurgery 30: 205-209.

18. Jeong H, Kim J, Choi HS (2011) Changes in integrity of normalappearing whit matter in patients with moyamoya disease: A diffusiontensor imaging study. Am J Neuroradiol 32: 1893-1898.

19. Lehman CD (2012) Diffusion weighted imaging ( DWI) of breast: Ready forclinical practice. Eur J Radiol 81: 80-81.

20. Tang ML, Chen X, Chen ZY (2014) The manifestation ofamyotrophic lateral sclerosis on 3.0T MRI and diffusion tensortracking. J Jinan University 35: 555560.

21. Nitkunan A, Barrick TR, Charlton RA (2008) Multimodal MRI in cerebral small vessel disease: Its relation-ship with cognition and sensitivity to change over time. Stroke 39: 1999-2005.

22. Moorhouse P, Rockwood K (2008) Vascular cognitive impairment: Current concepts and clinical development. Lancet Neurol 7: 246-255.

23. Tekina S, Cummings JL (2002) Frontal-subcortical neuronal circuits and clinical neuropsychiatry: An update. J Psychosom Res 53: 647-654.

24. Zhang Y, Schuff N, Jahng GH (2007) Diffusion tensor imaging of cingulum fibers in mild cognitive impairment and alzheimer disease. Neurol 68: 13-19. 